Annales Geophysicae (2003) 21: 615-626 (c) European Geosciences Union 2003

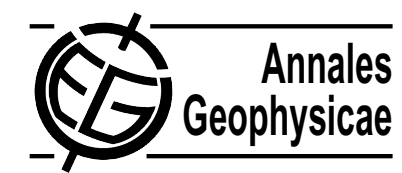

\title{
Photon path length distributions for cloudy skies - oxygen A-Band measurements and model calculations
}

\author{
O. Funk and K. Pfeilsticker \\ Institut für Umweltphysik, University of Heidelberg, Germany \\ Received: 1 November 2001 - Revised: 1 July 2002 - Accepted: 17 July 2002
}

\begin{abstract}
This paper addresses the statistics underlying cloudy sky radiative transfer (RT) by inspection of the distribution of the path lengths of solar photons. Recent studies indicate that this approach is promising, since it might reveal characteristics about the diffusion process underlying atmospheric radiative transfer (Pfeilsticker, 1999). Moreover, it uses an observable that is directly related to the atmospheric absorption and, therefore, of climatic relevance. However, these studies are based largely on the accuracy of the measurement of the photon path length distribution (PPD). This paper presents a refined analysis method based on high resolution spectroscopy of the oxygen A-band. The method is validated by Monte Carlo simulation atmospheric spectra. Additionally, a new method to measure the effective optical thickness of cloud layers, based on fitting the measured differential transmissions with a 1-dimensional (discrete ordinate) RT model, is presented. These methods are applied to measurements conducted during the cloud radar intercomparison campaign CLARE'98, which supplied detailed cloud structure information, required for the further analysis. For some exemplary cases, measured path length distributions and optical thicknesses are presented and backed by detailed RT model calculations. For all cases, reasonable PPDs can be retrieved and the effects of the vertical cloud structure are found. The inferred cloud optical thicknesses are in agreement with liquid water path measurements.
\end{abstract}

Key words. Meteorology and atmospheric dynamics (radiative processes; instruments and techniques)

\section{Introduction}

The deposition of the solar shortwave (SW) radiation is the driving force of many atmospheric processes, such as atmospheric dynamics or photochemistry. Thus, it is a primary factor controlling the climate of the globe (Stephens and

Correspondence to: O. Funk

(ofunk@montrouge.sema.slb.com)
Tsay, 1990; Wild et al., 1995, 1996, and others). Despite its important role for the terrestrial climate, the amount of $\mathrm{SW}$ radiation absorbed in the clear and cloudy atmosphere is still poorly understood. Under cloudy skies, the poor understanding is primarily due to either (a) deficits in understanding atmospheric absorber characteristics (interstitial aerosol absorption, water vapor line strength and continuum absorption or yet unknown absorption mechanism, such as water dimers) and (b) deficits in understanding the cloudy sky radiative transfer (e.g. results presented at the recent Chapman Conference on Atmospheric Absorption and Solar Radiation, AGU 2001).

The present paper explores in more detail possibility (b) by presenting joint cloudy sky photon path length distribution and cloud optical thickness measurements, cloud structure data, and model calculations. The cloudy sky photon path length distribution measurements were performed by means of high resolution oxygen A-band measurements in zenith scattered skylight. This type of measurement has been developing rapidly in recent years (e.g. Harrison and Min, 1997; Pfeilsticker et al., 1998). Historically, cloudy sky photon path length distributions have been shown to be approximately gamma distributed in a homogenously (and in the vertical inhomogeneously) scattering atmosphere (Van de Hulst, 1980; Marshak et al., 1995). From the very beginning it was clear that scattering problems in real cloud covered atmospheres may lead to far more complicated PPDs since cloud covers tend to appear in patches, multilayers or even as fractal cloud decks (e.g. Davis et al., 1996). Thus, real scattering atmospheres require assumptions on the distribution of the individual step sizes, which are different from the classical assumption of a well-defined exponential distribution, the latter leading to the classical diffusion assumption for homogenous, optically thick media. Clearly, real atmospheres do not fulfill this assumption, while theoretically they are more likely to support scattering statistics with individual step size distributions which allow for more frequent extreme step sizes. This lack of theoretical correctness in classical cloudy sky RT modelling led Davis and Marshak 
(1998) to suggest Levy type diffusion, characterized by individual step size distributions decreasing according to power laws (in contrast to the exponential distribution in Gaussian diffusion), to be more appropriate in cloudy sky scattering phenomena, a suggestion experimentally supported for many complicated cloudy sky cases by Pfeilsticker (1999) or recently by Min et al. (2001). Previous oxygen A-band PPD measurements, however, were suffering from several limitations, such as a limited temporal and, hence, spatial resolution at the expense of a high spectral resolution, an insufficient spectral retrieval technique, and a lack of validation. While overcoming the first limitation requires more sensitive instrumentation, being presently developed, the other limitations are addressed by the methodic improvement presented in this paper.

\section{Photon path length distributions, Beer's law and the Laplace transformation}

The basic idea of cloudy sky geometrical path length distributions is that the sequence of scattering events form a light path from which finally, only the total geometrical length is relevant. Extinction can then be regarded solely as gaseous absorption along this path. Since multiple scattering is a stochastic process, each photon will travel a different path. The probability that a photon has travelled a total path length $l$ is given by the probability density function (PDF) of the geometrical path lengths $p(l)$ (short PPD).

$p(l) d l=P\left(l-\frac{1}{2} d l<L<l+\frac{1}{2} d l\right)$

This distribution is only valid in absence of absorption, i.e. for the scattering processes only. In an absorbing atmosphere, the transmission with respect to absorption, described by the absorption cross section $\sigma$ and the number concentration $n$, along a specific path of length $L$ is given by Beer's law.

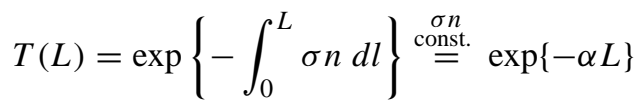

For constant extinction $\alpha=\sigma n$ the total transmission of all occurring paths is given by the integral over the path length distribution weighted with the Beer factor $\exp \{-\alpha l\}$.

$T(\alpha)=\int_{0}^{\infty} p(l) \exp \{-\alpha l\} d l=\mathcal{L}_{\alpha \leftarrow l} p(l)=\tilde{p}(\alpha)$

This expression is formally equivalent to the Laplace transformation $\mathcal{L}$ of $p(l)$ with respect to $\alpha$. Thus, it is a consequence of the exponential form of Beer's law that $T(\alpha)$ is the Laplace transform of the PPD. This relationship implies that the explicitly unmeasurable path length distribution can be derived by measuring the transmissions for different absorption strengths and inverting the Laplace transformation. Techniques for this non-trivial inversion are discussed in Sect. 3.
The above relation is only valid for constant extinction, i.e. in an isotropic atmosphere. In the real atmosphere mainly the pressure and temperature dependencies of $n$ and $\sigma$ introduce a vertical anisotropy. For a general atmosphere the path integral in the exponent of Eq. (2) can be different for each photon trajectory. For an ensemble of $N$ photons the total transmission $\bar{T}$ is then given by the ensemble average:

$\bar{T}=\frac{1}{N} \sum_{i=1}^{N} \exp \left\{-\int_{0}^{L_{i}} \sigma_{i}(l) n_{i}(l) d l\right\}$.

The right-hand side of this equation is highly complex, since it requires the knowledge of each photon trajectory. Moreover, $\bar{T}$ cannot be expressed as an explicit function of $\alpha$. The Laplace transformation of $p(l)$ is, therefore, in general not given by the transmissions $T$ as in Eq. (3). Equation (4) can, however, be simplified approximately using $p(l)$ and a mean absorption coefficient $\bar{\alpha}$.

$\overline{T\left(\alpha_{i}, L_{i}\right)} \approx T(\bar{\alpha})=\int_{0}^{\infty} p(l) \exp \{-\bar{\alpha} \cdot l\} d l$

This approximation introduces a systematic error, but its approximated validity can be tested by a Monte Carlo simulation of the photon paths in a non-isotropic atmosphere (see Sect. 4).

\section{Inversion of the Laplace transformation}

The possibility ofretrieving the path length distribution from measured transmissions is based on an accurate technique for the calculation of the inverse Laplace transformation. This inversion is not straightforward. Direct techniques are unsuited (see Appendix A). Therefore, a constrained method imposing a priori assumptions on the analytical form of the path length distribution can be used. Most common in this context is the Gamma distribution. Van de Hulst (1980, 17.2.3) shows that the photon optical path distribution for homogeneous slabs can be well approximated by Gamma distributions. Marshak et al. (1995) show that the distribution of the photon displacement at the point of escape from homogeneous, as well as horizontally fractal cloud layers, is well approximated by Gamma distributions. The Gamma distribution (Eq. (6)) has two parameters, $\langle l\rangle$ and $\kappa$, each being functions of the first two moments of the PDF.

$p(l)=\frac{1}{\Gamma(\kappa)\left(\frac{\langle l\rangle}{\kappa}\right)^{\kappa}}(l)^{\kappa-1} e^{-\frac{\kappa l}{\langle l\rangle}}, \quad$ with $\quad \kappa=\frac{\langle l\rangle^{2}}{\operatorname{var}(l)}$

The inversion problem is solved if the Laplace transformation of the analytical expression of the PDF can be calculated. For the Gamma distribution it is given by:

$\tilde{p}(k)=\frac{1}{\left(1+\frac{\langle x\rangle}{\kappa} k\right)^{\kappa}}$.

A more general, numerical method is based on accurate forward modelling of the measured transmissions according 
Table 1. Scattering characteristics used for the radiative transfer models

\begin{tabular}{lll}
\hline Scattering events & Cross section/extinction & Phase function \\
\hline Rayleigh & $\sigma_{R S}=4.02 \times 10^{-28} / \lambda^{4.04} \mathrm{~cm}^{2}$ (Nicolet, 1984) & $\psi(\cos \vartheta)=\frac{3}{4}\left(1+\cos ^{2} \vartheta\right)$, no polarization \\
Aerosol & LOWTRAN Spring/Summer, background (Isaacs et al., 1987) & Henyey-Greenstein: $\psi(\cos \vartheta)=\frac{1-g^{2}}{4 \pi\left(1+g^{2}-2 g \cos \vartheta\right)^{3 / 2}, g=0.7}$ \\
Cloud & homogeneous, exponential step size distribution $\langle s\rangle=H_{c} / \tau_{c}$ & Henyey-Greenstein, $g=0.85$ water clouds, $g=0.7$ ice clouds \\
Ground & albedo 0.3 & $\psi(\cos \vartheta)=2 \cos \vartheta$, Lambertian \\
\hline
\end{tabular}

to Eq. (3). In this case any parameterized expression using parameters $p_{i}$ can be used as $\operatorname{PDF}, p(l)=f\left(l, a_{1}, \ldots, a_{n}\right)$. The parameters are then retrieved by applying a least-squares fit of observed (measured or from radiative transfer models) and forward modelled transmissions. Another well suited standard distribution is the log-normal distribution:

$p(l)=\frac{1}{S l \sqrt{2 \pi}} \exp \left\{-\frac{(\ln l-M)^{2}}{2 S^{2}}\right\}$,

with parameters $M$ and $S$.

The validity of these PDF models is tested with a Monte Carlo model (see Sect. 4). For further analysis this numerical method with Gamma and log-normal distributions as models for $p(l)$ are used. Additionally, two modifications of $p(l)$ are made for practical purposes:

- Variable transformation

The path length is measured in units of vertical paths through the atmosphere. This is done by changing the independent variable from $\alpha$ to the vertical optical density $V O D=\alpha \cdot l_{\text {ver }}$. This changes the independent variable of $\tilde{p}(l)$ from $l$ to $l^{\prime}=l / l_{v e r}$. Since $\alpha$ is not constant in the atmosphere, $\operatorname{VOD}=\int_{0}^{\infty} \alpha(z) d z$ is used instead.

- Shift of the distribution

Since lengths of path shorter than one vertical path through the atmosphere cannot occur for transmission, the distribution is shifted by one vertical path by $l^{\prime}=$ $l-l_{\text {ver }}$ in old, or $l^{\prime}=l-1$ in new variables. Application of the translation property of the Laplace transformation yields a factor of $e^{-V O D}$ in the Laplace transform (see Eq. (A3))

\section{Validation with a Monte Carlo radiative transfer model}

The method is validated by modelling the path length distributions and transmissions for the atmosphere by a Monte Carlo radiative transfer model. Since the inaccuracy of the approximation in Eq. (5) strongly depends on the cloud situation, i.e. mainly the vertical extent and vertical variability of the paths, two different typical cases are presented here:
Table 2. Results (moments $m_{1 / 2}$ ) of the PPD retrievals for the first two moments using both PDF models for both cases. Also included are the results of the direct fit to the MC PDF, see Fig. 1

\begin{tabular}{clrrrrrr}
\hline Case & PDF model & \multicolumn{2}{c}{ Transmissions } & \multicolumn{2}{c}{ PDF direct } & \multicolumn{2}{c}{ MC model } \\
& & $m_{1}$ & $m_{2}$ & $m_{1}$ & $m_{2}$ & $m_{1}$ & $m_{2}$ \\
\hline 1 & Gamma & 11.7 & 177 & 12.3 & 185 & 13.4 & 251 \\
1 & log-normal & 12.0 & 196 & 13.5 & 258 & 13.4 & 251 \\
2 & Gamma & 4.6 & 33 & 3.7 & 19 & 4.8 & 42 \\
2 & log-normal & 4.7 & 39 & 4.5 & 37 & 4.8 & 42 \\
\hline
\end{tabular}

- Case 1: extended cumulus cloud cloud base $1000 \mathrm{~m}, \Delta H=3000 \mathrm{~m}, \tau_{c}=200$, $\lambda_{\text {Mie }}=15 \mathrm{~m}, g=0.85$

- Case 2: low level stratus cloud with thin cirrus cloud base $1000 \mathrm{~m}, \Delta H=1000 \mathrm{~m}, \tau_{c}=50$, $\lambda_{\text {Mie }}=20 \mathrm{~m}, g=0.85$ cloud base $7000 \mathrm{~m}, \Delta H=500 \mathrm{~m}, \tau_{c}=5$, $\lambda_{\text {Mie }}=100 \mathrm{~m}, g=0.7$

with $\Delta H$ being the vertical cloud layer extent and $\lambda_{\text {Mie }}$ the mean free path for Mie scattering within the layer.

The Monte Carlo model generates the photon trajectories by simulating the elementary scattering processes listed in Table 1. A backward approach is used to save computation time. The photon is injected into the atmosphere at the detector position in zenith viewing direction. It is then traced along its path. Upon escape from the model atmosphere, the probability for the last scattering to result in the direction towards the Sun is calculated, and a statistical weight equal to this probability is assigned to the trajectory. In order to simulate the transmission, the optical densities at 15 wavelengths, located in the centers of oxygen A-band lines, are accurately integrated along the trajectories. For that to occur, the absorption cross section and number concentration are calculated according to a temperature profile taken from a radio sonde for a real measurement (see Sect. 5), the pressure is assumed to follow an exponentially decreasing profile. Additionally, the vertical optical density is integrated. The parameters for the PDF model (Gamma or log-normal) are then calculated by a least-squares fitting of the transmis- 

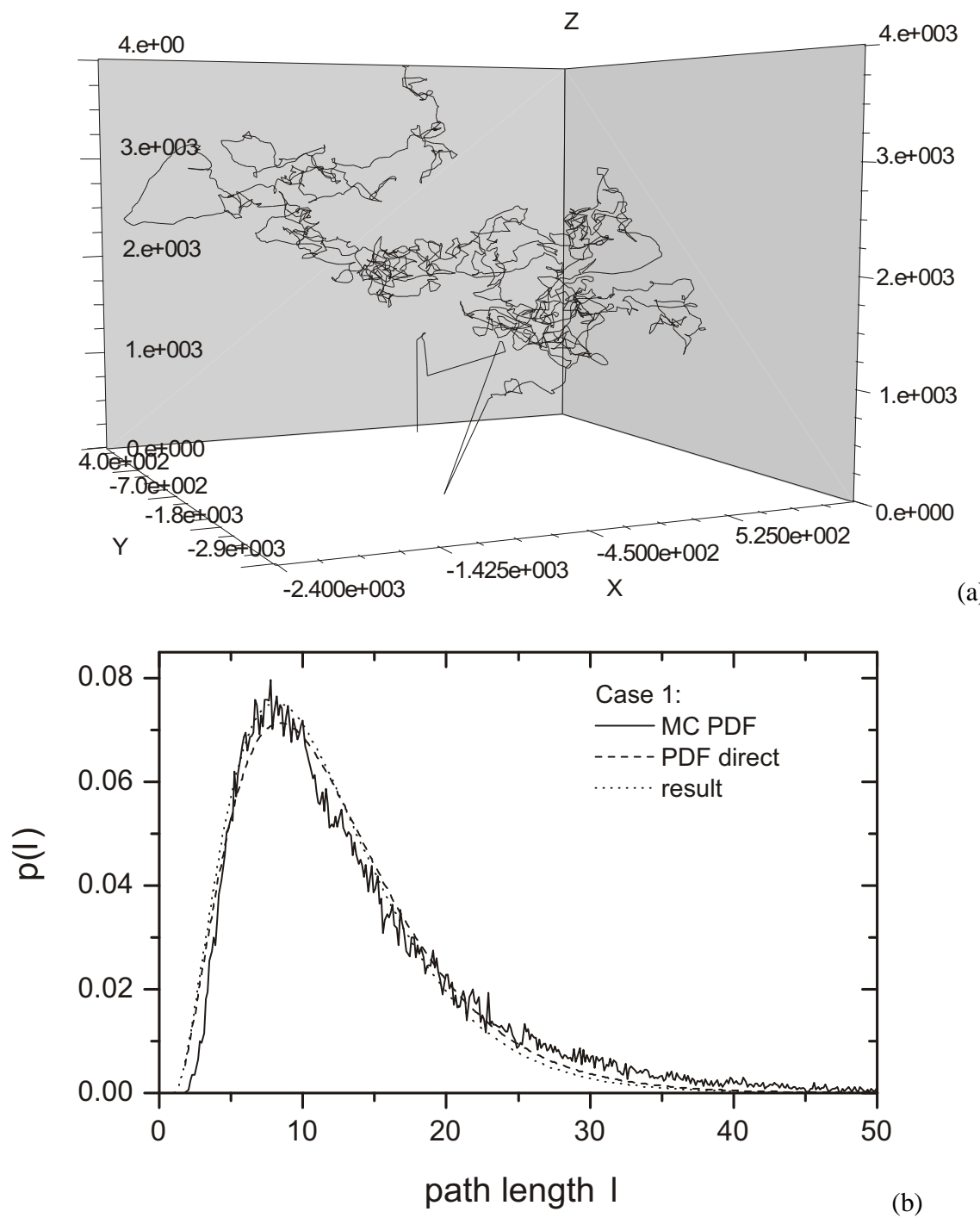

Fig. 1. The MC path length distributions and validation results for the two cases. Panels (a) and (c) show exemplary photon trajectories for the Cases 1 and 2 discussed in Sect. 4, respectively. The atmosphere is cut at the cloud top, axis units are in m. While panel (a) shows the photon diffusion in the extended cloud layer, reflection between the two cloud layers can be seen in panel (c). The panels (b) and (d) show the resulting path length distributions for 1 million photons. Path lengths are given in units of the vertical path. Panel (b) shows a typical broad distribution, while the reflection between the two layers generate a bimodal distribution for Case 2. To fit these PDFs, a Gamma distribution is used for Case 1 (b) and a log-normal distribution is used for Case 2 (d). Included is the results of a direct fit of the Monte Carlo PDF with the PDF model (labelled as PDF direct). This comparison demonstrates the validity of the PDF model. Good agreement is found for Case 1; however, the Gamma function cannot follow exactly the rising flank which starts at higher path lengths and is steeper. The single peaked PDF model does not match the two modes of the distribution for Case 2. Finally, the results of the retrieval, using the modelled transmissions, is included (labelled as result). For Case 1 the result is similar to the PDF direct result. For Case 2 there is quite a difference between the two retrieved log-normal distributions. The result from the fit with the transmissions looks closer and the moments, see Table 2 , are better matched than for the PDF direct fit, which is "misled" by the bimodal form (continues next page).

sions resulting from the RT model $\left(T_{M C}\right)$ with those calculated from the parameterized PDF model (discrete in $l$ with $i$ points, correctly normalized) for all $V O D s$ :

$T_{M C}(V O D) \stackrel{L S F}{=} \sum_{i=1}^{N} p\left(l_{i},\left\{a_{j}\right\}\right) \exp \left\{-V O D \cdot l_{i}\right\}$

The results are shown in Fig. 1 and Table 2. For Case 1 the path lengths are underestimated by approx. 10-15\% using the log-normal and Gamma distribution. Also, the sec- ond moment is underestimated substantially. For the Gamma distribution this is a consequence of the fixed shape of the distribution, as can be seen in Fig. 1b. It rises too fast for path lengths just above 1, while it decreases too fast around path lengths of 30 . The log-normal model gives excellent results for the direct fit (Table 2) but similar results as the Gamma distribution from the transmission fit.

For Case 2 the log-normal model fits the moments best. Excellent agreement is found, indicating that atmospheric in- 


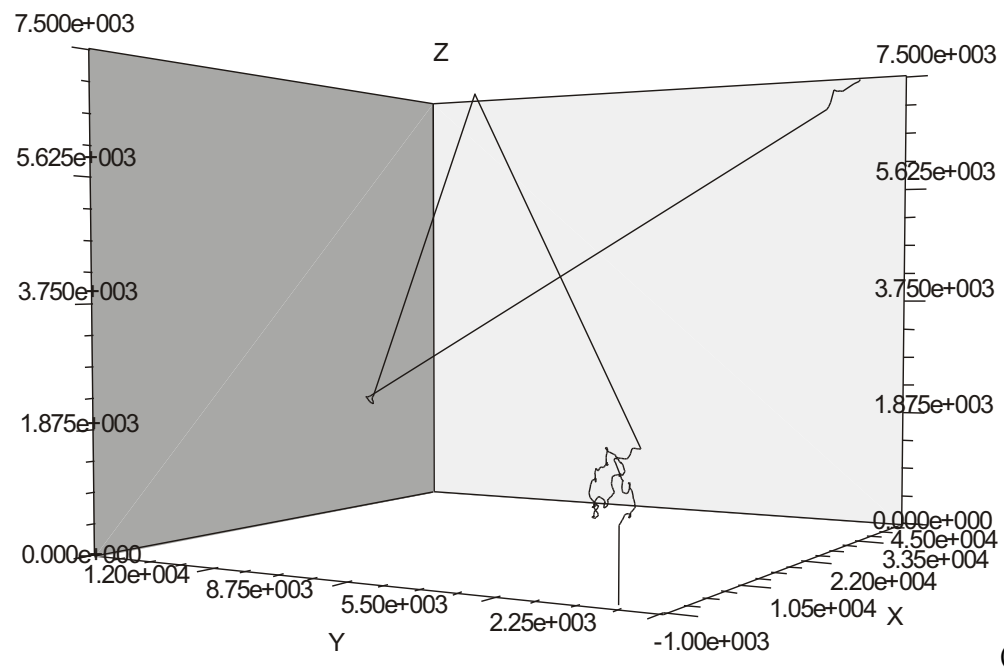

(c)

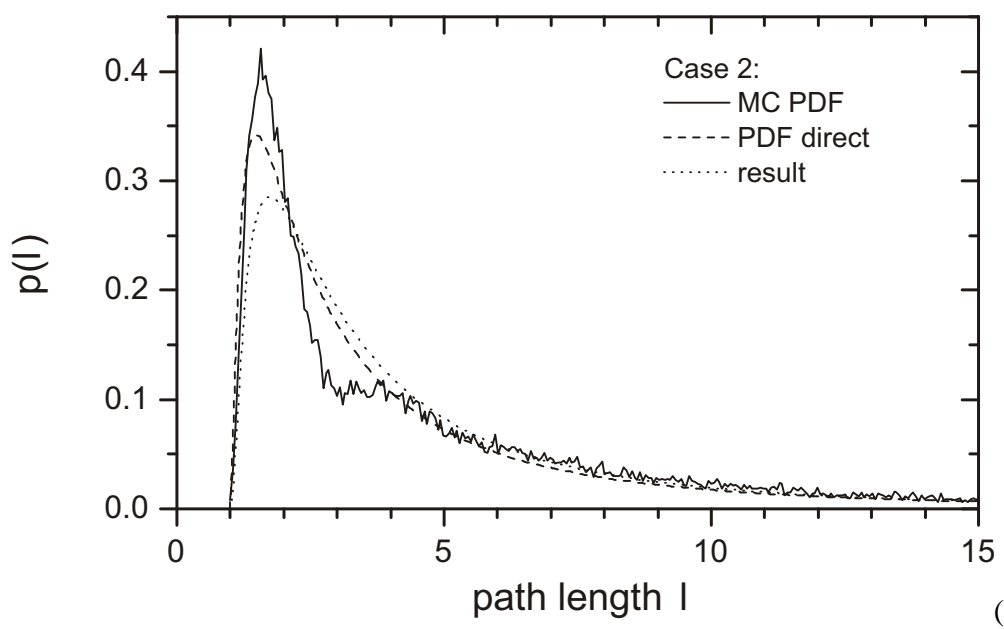

(d)

Fig. 1. ... continues

homogeneity concerning temperature and pressure does not play a critical role, and Eq. (5) is a valid approximation. In this case the transmission fit results in even better agreement in the moments than the direct fit (see Fig. (1d).

To summarize this validation, it can be said that the choice of the PDF model appears to be more critical than the approximation made for the isotropy. Even though the log-normal distribution appears to be the better choice in all cases, in practice often the Gamma distribution is used due to a more stable convergence behavior in the fit (see Sect. 5).

\section{Spectrum analysis and instrumental setup}

The transmissions for a wide range of absorption strengths $T(\alpha)$, i.e. the Laplace transform of the path length distribution (Eq. 3), can be measured by oxygen A-band spectroscopy (e.g. Pfeilsticker et al., 1998; Harrison and Min, 1997). The main advantages of the oxygen A-band $\left(b^{1} \Sigma_{g}^{+} \leftarrow X^{3} \Sigma_{g}^{-}, 759-775 \mathrm{~nm}\right)$ are the lack of interference with other atmospheric absorbers (as oxygen is the only ab- sorber in this spectral range) and the constant oxygen mixing ratio. Additionally, the absorption characteristics of oxygen are known very accurately. The key problem in the spectrum evaluation is the insufficient resolution of the spectrograph. Since the true transmissions are required for the analysis of Eq. (3), the measured spectrum must be corrected for resolution effects. Pfeilsticker et al. (1998) corrected the transmissions for the centers of a set of A-band absorption lines by using a curve of growth measured for direct light. However, the curve of growth is dependent on the path length distribution, since it modifies the line shapes of the absorption lines. Therefore, this method introduces a systematical error. The refined method presented here solves this problem by first modelling the true absorption line shapes for the current path length distribution at high spectral resolution $\left(5 \times 10^{-2} \mathrm{pm}\right)$ and then converting the whole modelled spectrum to the measurement resolution by convolution with the instrument function (see Fig. (4). The spectroscopic model uses spectroscopic data from Ritter and Wilkerson (1987) and Gamache et al. (1998), and radio sonde atmospheric pressure and temperature profiles, $p$ and $T$, to calculate cross section spec- 


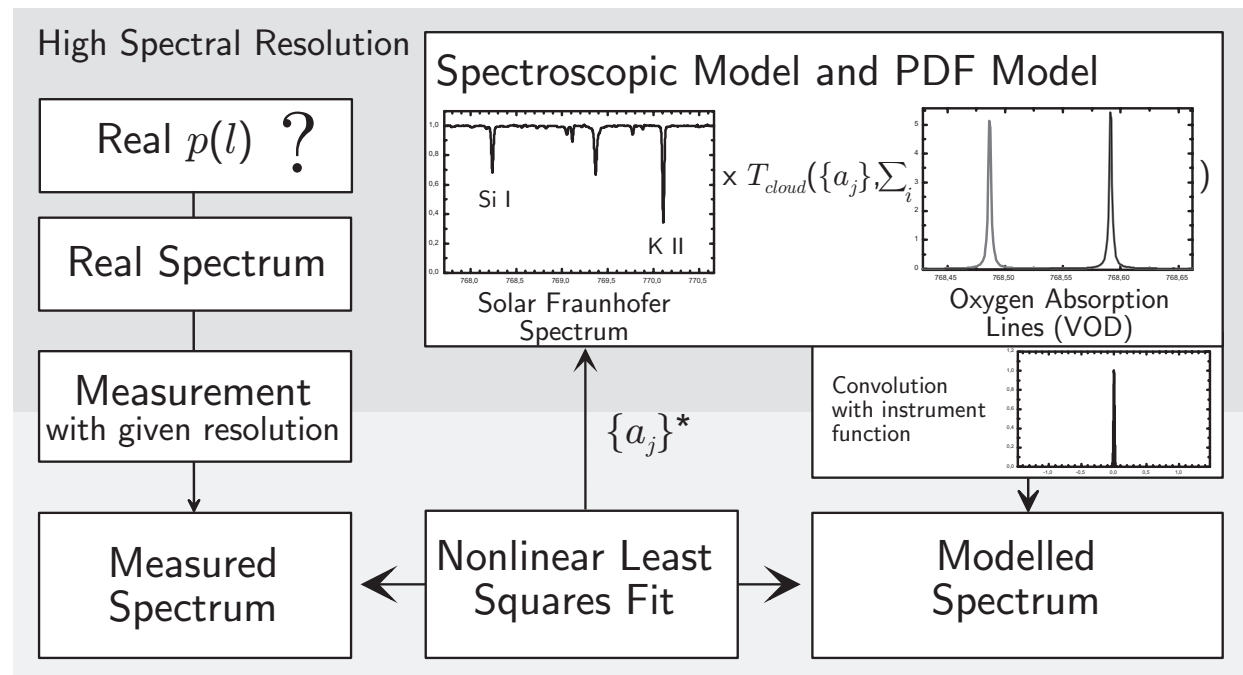

Low Spectral Resolution

Fig. 2. Schematic view of the spectrum evaluation process. The critical spectral calculations are performed at high spectral resolution (for 100000 discrete wavelengths). The fit optimizes the parameters of the PDF model $\left\{a_{j}\right\}$. The spectroscopic model contains a Fraunhofer reference. Additionally, terrestrial absorption above the cloud is separated from the actual cloud transmission, see Eq. (11). The convolution to measurement resolution is a critical step. Any inaccuracy in the instrument function will be compensated for by an erroneous modification of the PPD. The accuracy of the evaluation can be tested for direct light measurements, where a delta type PPD is expected.

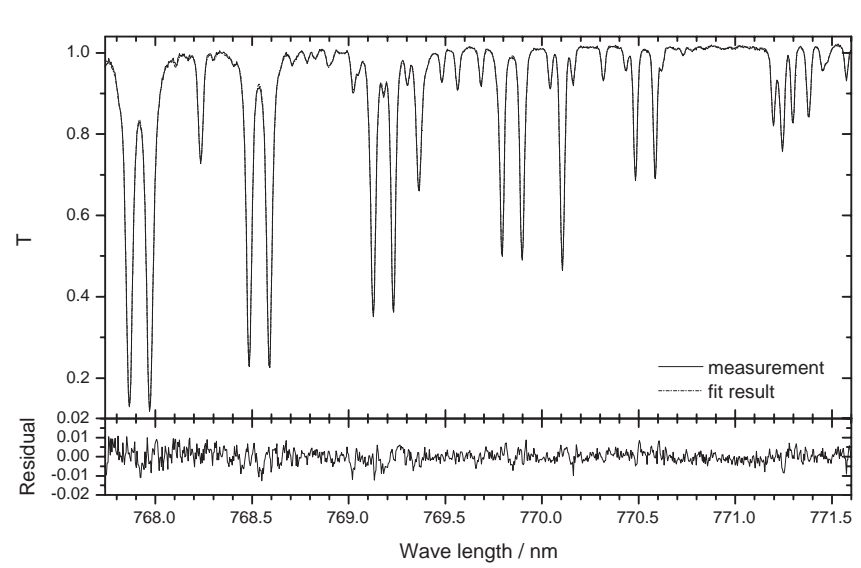

(a)

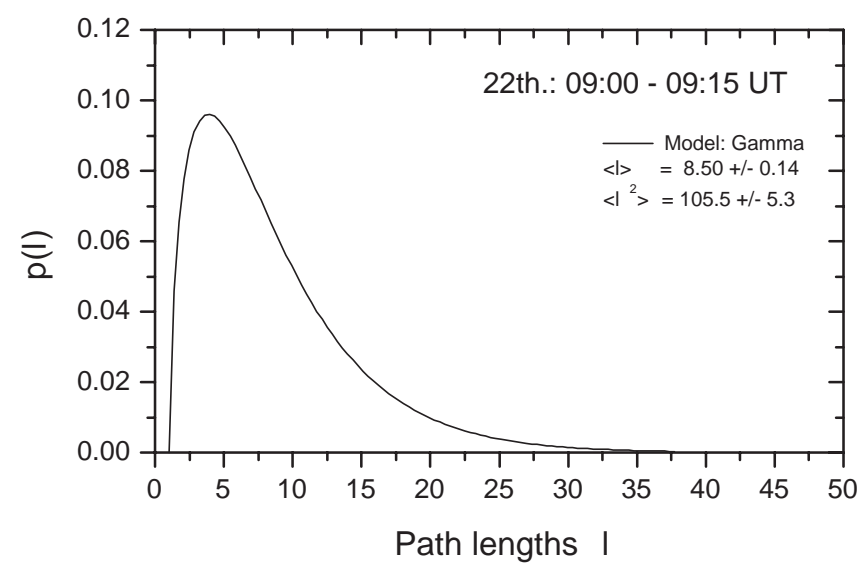

(b)

Fig. 3. (a) A sample for a measured and fitted spectrum with a typical residual. The oxygen spectrum consists of pairs of rotational lines. Also contained are strong Fraunhofer lines, e.g. at $768.2 \mathrm{~nm}$ (Si I) and $770.1 \mathrm{~nm}$ (K II). (b) The retrieved path length distribution corresponding to the spectrum above. A Gamma distribution is used as PDF model. $1 \sigma$ errors are included.

tra, $\sigma_{i}(T, p, \lambda)$. Voigt line profiles and temperature corrected line strengths are used for the individual rotational absorption lines, $i$, for 40 atmospheric layers, $l$. The light path above the cloud is assumed to be straight with a incidence angle $\mu_{0}(=\cos (S Z A)$, with the Sun zenith angle $S Z A)$. Absorption above the cloud top can, therefore, be separated from absorption occurring inside the cloud and below (scattering above the cloud top is neglected). Therefore, two references spectra, $R_{a / c}$ for above and below the cloud top, respectively, containing the vertical optical depth (VOD) of all absorption lines, are calculated by multiplication of the absorption cross section with the layer column density, $c_{l}$, and summing up over the corresponding layers (the layer containing the cloud top is added proportionally to both references).

$R_{a / c}(\lambda)=\sum_{i} \sum_{l} c_{l} \cdot \sigma_{i}\left(T_{l}, p_{l}, \lambda\right)$

The modelled high resolution spectrum is calculated by:

$I(\lambda)=\exp \left\{-\left(F(\lambda)+1 / \mu_{0} \cdot R_{a}(\lambda)\right)\right\} \cdot T_{\text {cloud }}(\lambda)$

with the Fraunhofer reference $F$ which accounts for spectral structures in the sunlight and the transmission below the 


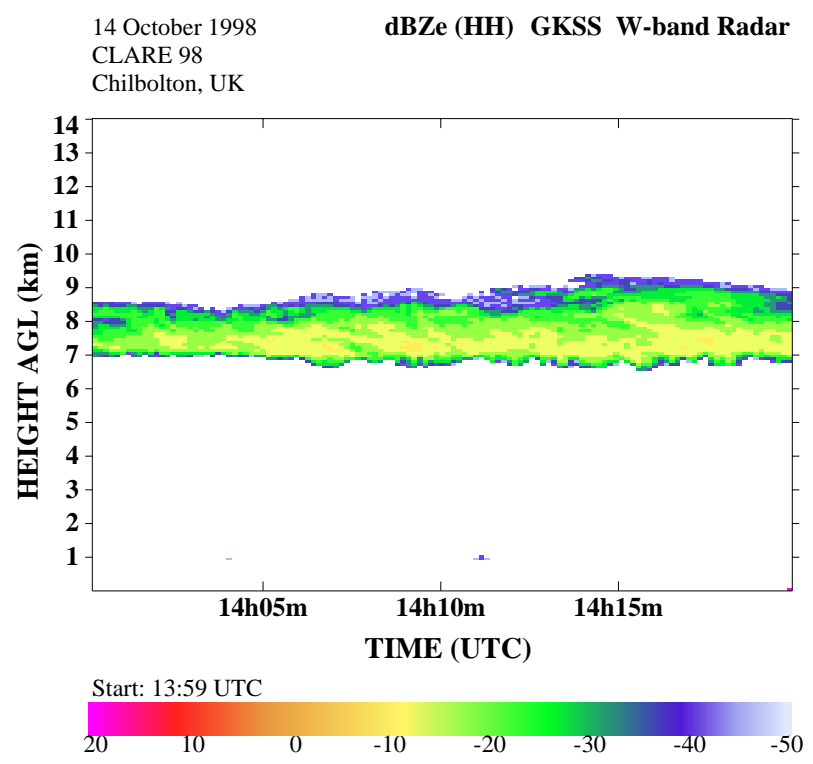

(a)

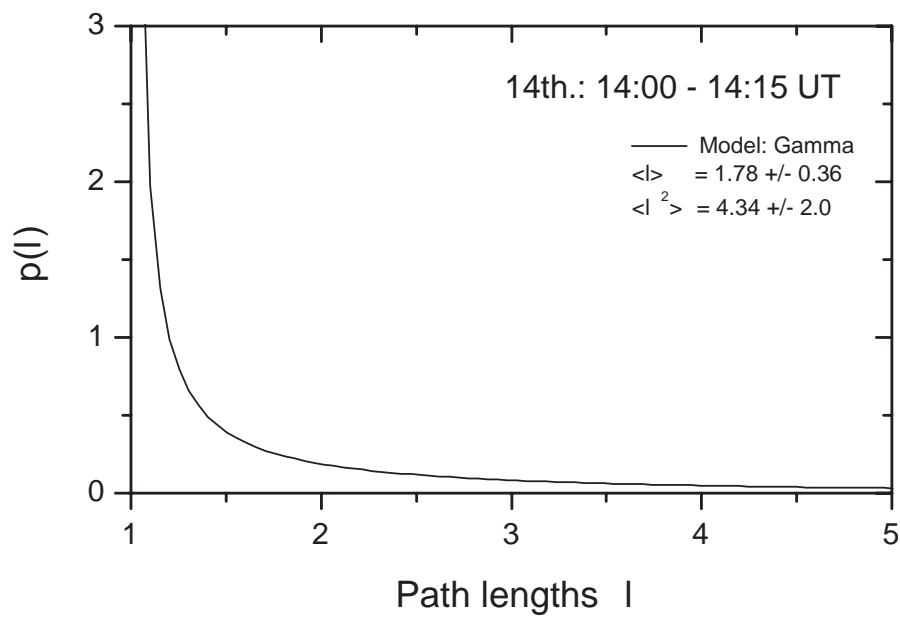

(b)

Fig. 4. (a) Cloud structure (backscatter ratio) measured by the $94 \mathrm{GHz}$ cloud Radar MIRACLE. (b) A high cirrus cloud layer reduces the mean photon path length compared to the clear sky, due to an increased probability for a nearly vertical path after a scattering event in high altitudes.

cloud top, $T_{\text {cloud }}$, calculated using a discrete and correctly normalized PDF model $p\left(l_{i}\right)$ with parameters $\left\{a_{j}\right\}$ :

$T_{\text {cloud }}(\lambda)=\frac{1}{N} \sum_{i=1}^{n_{\text {pdf }}} p\left(l_{i},\left\{a_{j}\right\}\right) \exp \left\{-l_{i} \cdot R_{c}(\lambda)\right\}$

This modelled spectrum is then convoluted $(\star)$ with the instrument function, $f_{\lambda}$, and fitted to the measured spectrum, $I^{\text {meas }}$, using a modified Levenberg Marquard method for the least-squares fit, (Moré et al., 1980), with the $\left\{a_{j}\right\}$ being the free parameters.

$I\left(\lambda,\left\{a_{j}\right\}\right) \star f_{\lambda} \stackrel{L S F}{=} I^{\text {meas }}(\lambda) \rightarrow\left\{a_{j}\right\}^{*}$

In the retrieved PDF the photon path lengths are given in units of the cloud top height.

The instrumental setup is equivalent to the setup described in Pfeilsticker et al. (1998). The sky light collected by a zenith pointing telescope (field of view $0.86^{\circ}$ ) is conducted by a quartz fiber via an optical band pass filter $(771.4 \mathrm{~nm}$, FWHM of $11.2 \mathrm{~nm}$ ) into an Echelle monochromator (Model MPP1, Aerolaser, focal length $1250 \mathrm{~mm}$, numerical aperture $\mathrm{f} / 15.3$, grating: blaze angle $65^{\circ}, 100 \mathrm{gr} / \mathrm{mm}$ ). The spectral resolution is $19.4 \mathrm{pm}$. The spectrum is recorded by a photo diode array detector (1024 channels, $25 \mu \mathrm{m}$, channel dispersion $4.1 \mathrm{pm}$ ), digitized (ADC resolution $16 \mathrm{bit}$ ) and stored on a PC. The raw spectrum is corrected for electronic offset, photo diode dark current and the overall spectral sensitivity. For further details on the measurements and evaluation, refer to Funk (2000).

Due to the low intensity of the zenith scattered light and the high spectral resolution of these measurements, the spectra must be integrated for typically 900 seconds. (This is mainly a constraint of the low gain of the PDA detector used, since modern CCD detectors are much more sensitive. However, the problem of low photon statistics remains.) This integration corresponds to an intensity-weighted temporal averaging. Under the assumption of slow changes in the cloud structure, the temporal averaging corresponds to a spatial averaging over a scale, determined by the integration time and the drift speed of the clouds (ergodicity). Additionally, the diffusion inside the cloud leads to spatial averaging. The size of this radiative smoothing scale (Marshak et al., 1995; Savigny et al., 1999) is strongly dependent on the degree of inhomogeneity. In the case of a single, homogeneous cloud layer, the mean lateral displacement for transmission is equal to the layer thickness. More details on scales are discussed in Pfeilsticker (1999).

A measured spectrum, together with the result of the evaluation and the retrieved path length distribution, is shown in Fig. 3.

\section{Cloud optical thickness}

A second result of the above fit is the high resolution transmission spectrum $T_{\text {cloud }}\left(\lambda,\left\{a_{j}\right\}^{*}\right)$. These transmissions can be compared to those resulting from radiative transfer model calculations. Here the 1-D discrete ordinate RT model DISORT (Stamnes et al., 1988) is used. The absorption properties $\left(\sigma_{a}\right)$ are constant and known. The scattering properties can be parameterized using the cloud optical thickness $\tau_{c}$, defined as the vertical integral of the scattering coefficient $\alpha_{s}$, $\tau_{c}=\int_{H_{c}} \alpha_{s} d z$, or as $\tau_{c}=H_{c} / \lambda_{c}$, with the mean free path inside the cloud $\lambda_{c}$ and the cloud geometrical thickness $H_{c}$. 

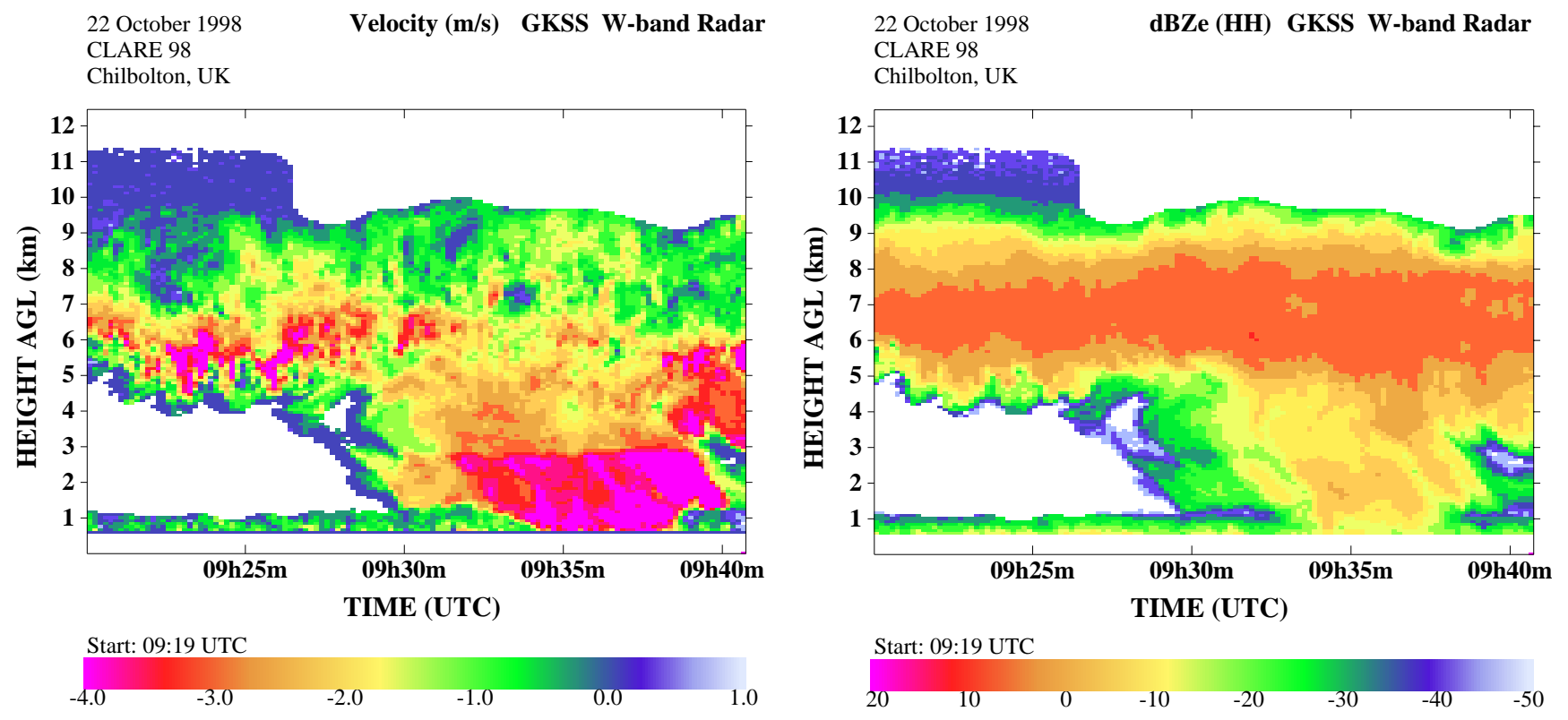

(a)

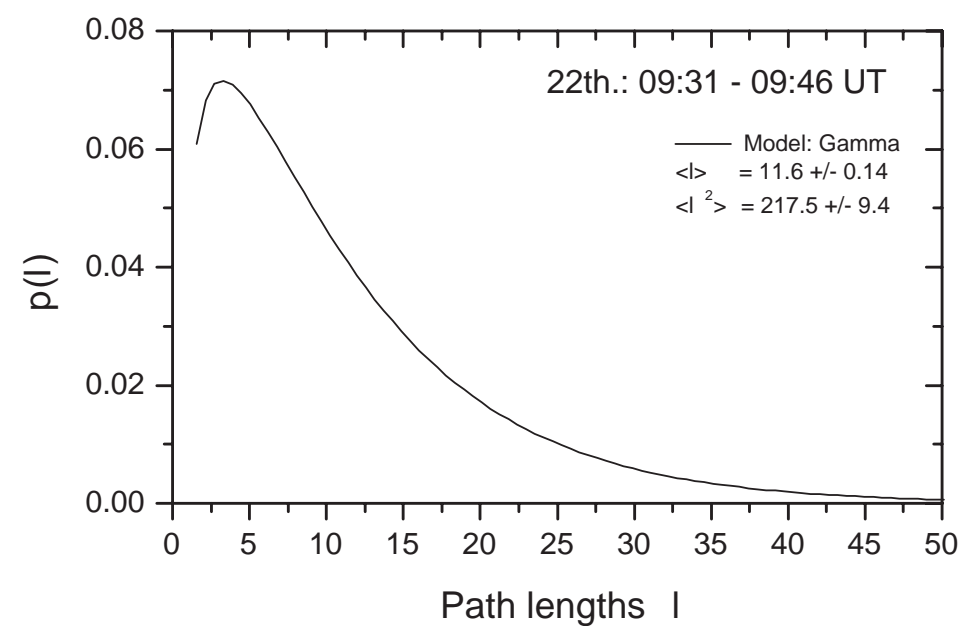

(b)

Fig. 5. (a), (c) Cloud structure (backscatter ratio) and vertical (Doppler) velocity measured by the $94 \mathrm{GHz}$ cloud Radar MIRACLE. In the first Case (a) two pronounced layers can be found, while no clear layer borders are present in the second Case (c). In this case the cloud fills the sky between $300 \mathrm{~m}$ and $10 \mathrm{~km}$ and is internally more inhomogeneous. (b), (d) The retrieved PPD for the two cases reflect the difference in cloud structure. The distribution is broader for the first Case (b), as expected for two cloud layers, see Fig. 6 (Fig. 5 continues next page).

All other scattering properties are given in Table 1. Several cloud layers can be included. The free model parameters are the cloud optical thicknesses $\left\{\tau_{c, i}\right\}$ of these layers. A leastsquares fit is used to find the $\left\{\tau_{c, i}\right\}^{*}$ for which the transmissions from the RT model agree best with those gained from the PPD retrieval.

$T_{\text {cloud }}(\lambda) \stackrel{L S F}{=} T^{R T}\left(\lambda,\left\{\tau_{c}\right\}, \overline{\sigma_{a}}\right) \rightarrow\left\{\tau_{c}\right\}^{*}$

Since the transmission at a certain wavelength is only depending on the path length distribution, this method yields the equivalent optical thicknesses of homogeneous cloud layers which result in a similar path length distribution. This optical thickness can be compared with the $\tau_{c}$ gained from liquid water path (LWP) measurements, using the effective radius $r_{e}$ for the corresponding cloud type (Slingo, 1989):

$\tau_{c}=\frac{3}{2} \frac{\mathrm{LWP}}{\overline{r_{e}}}$.

\section{Results}

The measurements presented here were conducted during the cloud RADAR intercomparison campaign CLARE'98 at Chilbolton, Hampshire, UK $\left(51.13^{\circ} \mathrm{N}, 1.43^{\circ} \mathrm{W}, 84 \mathrm{~m}\right)$ during October 1998. The collocated zenith viewing $94 \mathrm{GHz}$ cloud 

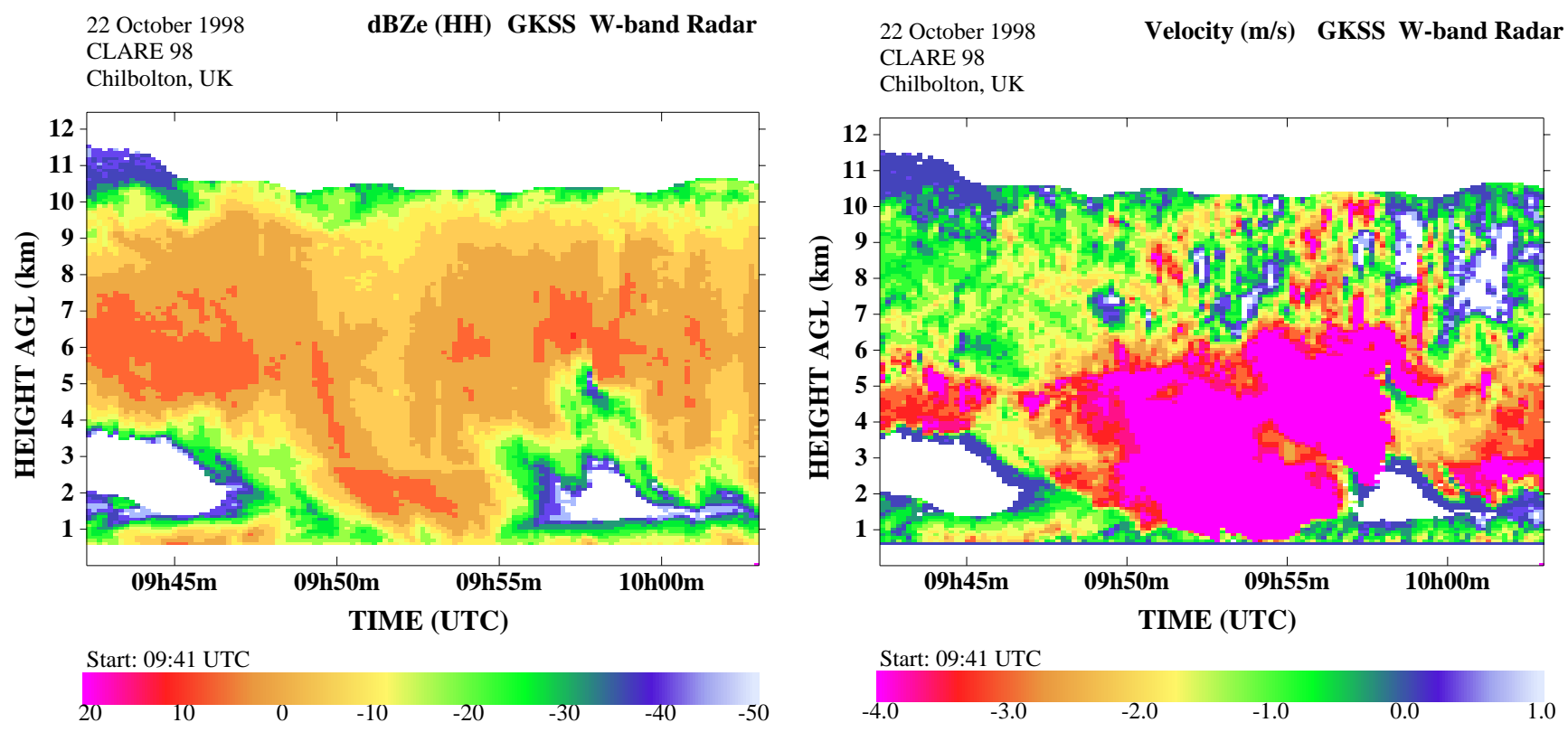

(c)

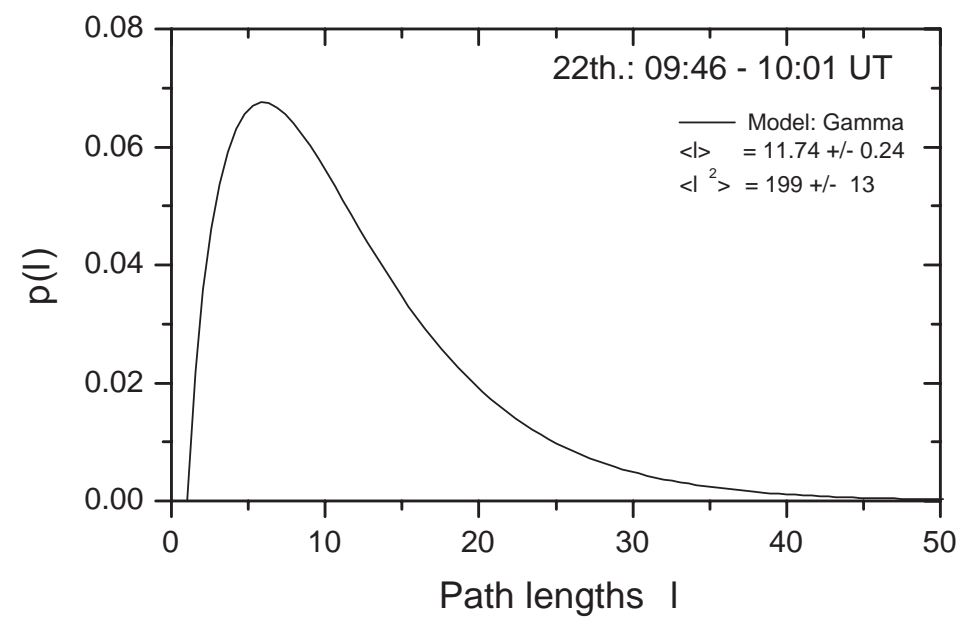

(d)

Fig. 5. ... continues

radar MIRACLE operated by GKSS (Geesthacht, Germany) recorded detailed information about the cloud structure. Of particular interest for this study is the vertical distribution of the cloud layers and the cloud top height. The cloud base is measured accurately by a ceilometer. Additionally, data collected by a microwave radiometer operated by TUE (Eindhoven, Netherlands) was used to retrieve the cloud liquid water path. The sum of these complementary measurements form a good data set to study the effect of cloud structure on the radiative transfer. Some exemplary cloud cases are presented here.

Figure 4 shows the result for a relatively thin cirrus cloud layer in about $8 \mathrm{~km}$ height. A high cloud layer is expected to reduce the mean photon path lengths for zenith viewing geometry, since the probability for a vertical path is largely increased. The direct light air mass factor $\left(\mathrm{AMF}=1 / \mu_{0}\right)$ is 2.4 for this measurement, and the retrieved mean path length is 1.8 (always in units of the vertical path), which agrees with the expectation. The exact form of the PPD is determined by the PDF model; however, a maximum probability for nearly vertical paths is reasonable for this case.

Figure 5 shows the cloud structures and retrieved PPDs for two subsequent measurements. During the first measurement (09:31-09:46 UT), two separated extended cloud layers are observed. The weak structure between these layers results from precipitation originating from the upper layer. Since the larger precipitating drops cause a superproportional large backscatter signal (the backscatter ratio depends on the particle diameter $d$ to the sixth $d^{6}$ ), the radar signal between the cloud layers around 09:35 UT is presumably caused by 


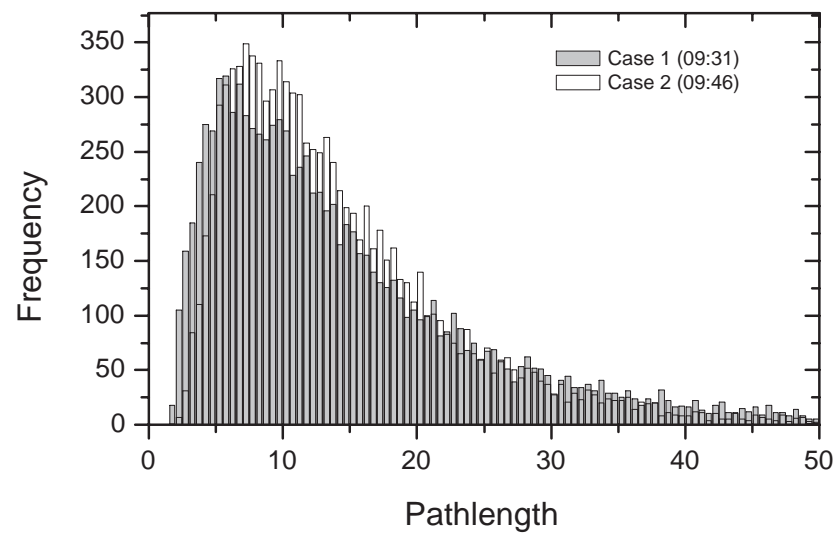

Fig. 6. Monte Carlo path length histograms calculated for the two measurements given in Fig. 5, using the cloud optical thicknesses from Table 3. The distributions show the same qualitative behavior as observed. The distribution is broader for the case of separated layers (1) compare to the case of one compact layer (2).

optically thin rain, Fig. 5a. During the second measurement (09:46-10:01 UT), heavy precipitation falls out of the upper layer, closing the gap between the two layers. An inhomogeneous mixture of cloud and rain emerges from the cloud base at $300 \mathrm{~m}$ (from ceilometer) to the cloud top at $9000 \mathrm{~m}$ with no clear borders, Fig. 5c. This transition from a two-layer stack to a single extended layer is reflected by the retrieved PPDs. While the mean path length remains nearly constant $\langle l\rangle=11.6$, the distribution is wider in the first case and more concentrated around the mean for the second case. This is also seen in lower second moments of $\left\langle l^{2}\right\rangle=217$ for the first distributions compared to $\left\langle l^{2}\right\rangle=199$ for the second case. The broadening of the distribution for two separated cloud layers can be explained by reflections between the cloud layers which dominate the radiative transfer in this case, while for a compact layer the photon path lengths are more centered around the diffusion mean path length.

This behavior is verified by Monte Carlo simulations for these two cases. Therefore, the optical thickness of the cloud layers was derived using the method described in Sect. 6 . The results are displayed in the left half of Table 3, marked as $\tau_{c, R T}$. Total cloud optical thicknesses of 144 and 197 are found for measurement 1 and 2, respectively. These optical thicknesses are used in the MC model described in Sect. 4, to generate path length histograms, Fig. 6. The measured shapes of the PPDs are qualitatively well reproduced by the MC model.

Finally, the retrieved cloud optical thicknesses are compared to the LWP data from the MW measurements. The measured mean LWPs for the two cases are $0.35 \mathrm{~mm}$ and $0.65 \mathrm{~mm}$, see Table 3. Using Eq. (15) and the retrieved cloud optical thicknesses, this results in reasonable values for the vertically averaged effective radii of $3.65 \mu \mathrm{m}$ and $4.96 \mu \mathrm{m}$ for Cases 1 and 2, respectively. Using the geometrical thickness and LWP and $r_{e}$ for standard cloud types reported by Stephens (1979) (Sc1, St1, St2), the agreement with the re-
Table 3. Results for the cloud optical thickness from the fit of the transmissions with the 1-D RT model (left half) and from the LWP measurements (right half). For the RT model, asymmetry factors of 0.85 and 0.7 were used for the lower and upper layer, respectively

\begin{tabular}{c|cc||ccc|c}
\hline Layer [m] & $\tau_{c, R T}$ & $\Delta \tau_{c, R T}$ & Type & $\begin{array}{c}\text { LWP } \\
{[\mathrm{mm}]}\end{array}$ & $\begin{array}{c}r_{e} \\
{[\mu \mathrm{m}]}\end{array}$ & $\tau_{c, M W}$ \\
\hline $09: 31$ & $\mathbf{1 4 4}$ & 17 & & $\mathbf{0 . 3 5}$ & 3.65 & 144 \\
\hline $300-900$ & 46 & 8 & Sc 1 & 0.28 & 9.91 & 42 \\
$5000-9000$ & 98 & 15 & St 1 & 0.22 & 4.21 & 71 \\
\hline $09: 46$ & $\mathbf{1 9 7}$ & 60 & & $\mathbf{0 . 6 5}$ & 4.94 & 198 \\
\hline $300-5000$ & 111 & 42 & Sc 1 & 0.66 & 5.4 & 183 \\
$5000-9000$ & 86 & 43 & St 2 & 0.2 & 4.21 & 71 \\
\hline
\end{tabular}

trieved optical thicknesses is not as good. However, this is not surprising given the variability of these parameters even for the same cloud type and the degree of inhomogeneity found, especially in the second case.

As a last case, Fig. 7 shows a single low level cumulus cloud layer between $300 \mathrm{~m}$ and $3300 \mathrm{~m}$. Typically for these clouds is that the cloud base and top are well-defined, while there is a great degree of internal inhomogeneity and large gaps. The retrieved optical thickness is $\tau_{c}=44.7 \pm 2.0$. The path length distribution resulting from the transmissions from the RT model is included in Fig. 7, together with the retrieved PPD. Both the PPDs agree within the error. A different shape, resulting from the plane-parallel model and the measurement, is not found for this case (within the errors), in agreement with findings reported by Portmann et al. (2001).

The mean LWP from the microwave radiometer is approx. $0.3 \mathrm{~mm}$. For a mean optical depth of 44.7, this LWP translates into a mean effective radius of $10 \mu \mathrm{m}$, which is a typical value for stratocumulus or cumulus clouds $(\mathrm{Sc} 2 / \mathrm{Cu}$, Stephens, 1979). However, the mean free path for the whole layer is $67 \mathrm{~m}$. This value is much longer than the typical mean free path of $10-15 \mathrm{~m}$ for $\mathrm{Sc} 2$ or $\mathrm{Cu}$ clouds. This enhanced mean free path might be explained by the inhomogeneous distribution of the liquid water content. For this case the cloud inhomogeneity only seems to prolong the mean free path, but has apparently no effect on the shape of the PPD.

\section{Conclusions}

With the presented improved method for oxygen A-band PPD measurements, a validated tool for the investigation of cloudy sky radiative transfer statistics is at hand. The analyzed cases already provide a taste of the new information that can be obtained, and the analysis which is possible provided an extensive data set, such as for CLARE'98. The effect of vertical cloud inhomogeneities on the path length distribution could be measured and reproduced qualitatively by Monte Carlo RT model calculations. Effective cloud optical thicknesses for equivalent horizontally homogeneous layers 
16 October 1998

CLARE 98

Chilbolton, UK

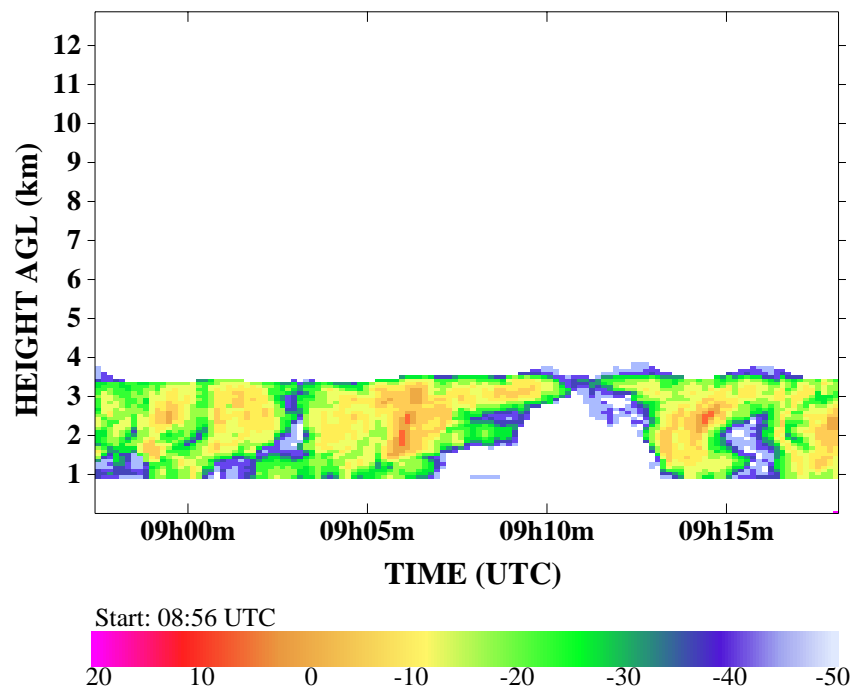

(a)

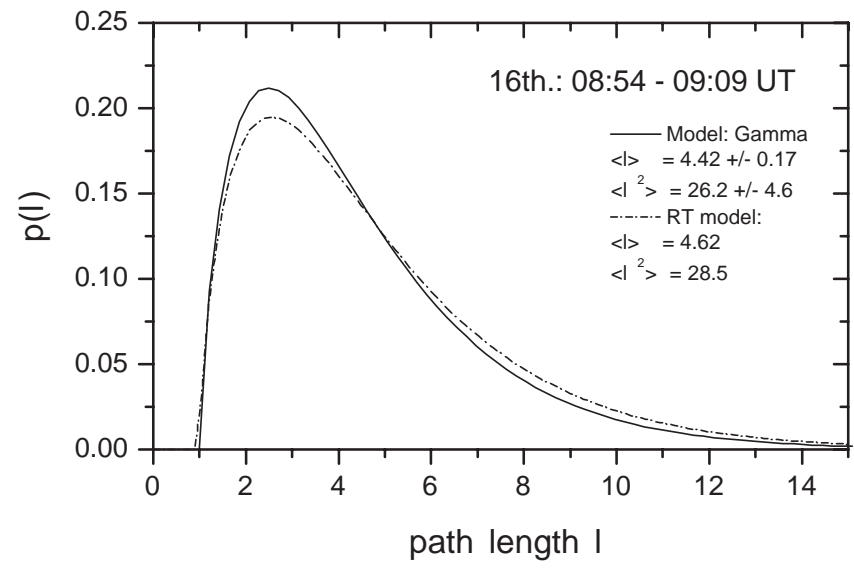

(b)

Fig. 7. (a) Cloud structure for an inhomogeneous cumulus cloud field. (b) PPDs derived from the measurement and from the 1-D RT model. Both distributions agree within the error.

were retrieved using a 1-dimensional (1-D) radiative transfer model. For the presented measurements, no significant difference between the measured and 1-D modelled PPDs could be observed, even for a horizontally inhomogeneous cloud cover. We argue that this is likely due to smoothing effects caused by the long averaging time, the main remaining limitation of the method. By increasing the temporal or spatial resolution to scales smaller than the radiative smoothing scale, the path length distribution measurements may help to unravel the statistics underlying the cloudy sky radiative transfer, i.e. whether and when it is a classical or anomalous (e.g. Levy-type) diffusion process.

Acknowledgements. This work was supported by the DFG (Deutsche Forschungsgemeinschaft) under the grant PF 384/1-1. We are grateful to M. Quante and O. Danne at GKSS for providing the cloud-radar images and Suzanne Jongen at TUE for the LWP data and ECMWF and UKMO for making available meteorological data.

Topical Editor J.-P. Duvel thanks a Referee for his help in evaluating this paper.

\section{Appendix A Direct Inversion of the Laplace transfor- mation}

The Laplace transform of a complex function $f(x)$ of the complex $\operatorname{argument} x$ is given by:

$\tilde{f}(k)=\mathcal{L}_{k \leftarrow x}\{f(x)\}=\int_{0}^{\infty} e^{-k x} f(x) d x$

if the integral converges. The main application of the Laplace transformation as a linear integral transformation lies in solving differ- ential equations by reducing the level of transcendence of the differential equation, see Bellman et al. (1966). A basic property of the Laplace transformation is the invariance under transformation both in $x$ and $k$ space.

$$
\begin{aligned}
\mathcal{L}\{f(x-s)\} & =e^{-s k} \mathcal{L}\{f(x)\} \\
\mathcal{L}\left\{e^{-t k} f(x)\right\} & =\tilde{f}(k+t)
\end{aligned}
$$

Two direct inversion techniques for the Laplace transformation are given here; however, neither of them is suited for the spectroscopic measurements.

\section{Complex Integration}

If $\tilde{f}(k)$ is known in the complex plane, the inversion of the Laplace transformation (back transformation) is given by the complex inversion formula:

$f(x)=\frac{1}{2 \pi i} \int_{C} \tilde{f}(k) e^{k x} d x$,

where $C$ is a carefully chosen contour. In particular, if $\tilde{f}(k)$ is known along a parallel to the imaginary axis, the inversion is given by:

$f(x)=\lim _{t \rightarrow \infty} \frac{1}{2 \pi i} \int_{c-i t}^{c+i t} \tilde{f}(k) e^{k x} d k$

if the limit converges. This formulation is known as the Bromwich integral. This method is applicable since only the real part of the Laplace transform is given by the measured transmissions. 


\section{Using the Characteristic Function}

The Characteristic Function $\chi$ of $p(l)$ is given by Fourier transform of $p(l)$. By expansion of the exponential function $\chi$ can be expressed by all moments $m_{j}$ of $p(l)$

$\chi(k)=\int_{0}^{\infty} p(l) e^{-i k l} d l=\sum_{j} \frac{(-i k)^{j}}{j !} m_{j}$

with $\quad m_{j}=\int_{0}^{\infty} \tilde{p}(\alpha) l^{j} d l$.

$p(l)$ is then given by the inverse Fourier transform:

$p(l)=\frac{1}{2 \pi} \int_{0}^{\infty} \chi(k) e^{i k l} d k$.

The moments $m_{j}$ can be found by expanding the exponential function in Eq. (3):

$T(\alpha)=\int_{0}^{\infty} p(l)\left(\sum_{j} \frac{(-\alpha)^{j}}{j !} l^{j}\right) d l=\sum_{j} \frac{(-\alpha)^{j}}{j !} m_{j}$.

For weak absorbers, i.e. $\alpha<l>\ll 1$, the first order approximation yields the well-known relation:

$T_{\alpha<l>\ll 1} \approx 1-\alpha<l>\quad$ and

$\tau=-\ln T \approx \alpha<l>$

This method uses the close relationship between Laplace transformation and Fourier transformation. Unfortunately, the inverse Fourier transformation is difficult to apply, since all moments are required. The complex $e$ function is converging very slowly with large oscillations for arguments $>1$.

\section{References}

AGU: Proceedings of the Chapmann Conference on Atmospheric Absorption of Solar Radiation, American Geophysical Union, Estes Park, Colorado, on August 13-17, 2001.

Bellman, R., Kalaba, R. E., and Lockett, J. A.: Numerical Inversion of the Laplace Transform: Applications to Biology, Economics, Engineering, and Physics, American Elsevier Publishing, New York, 1966.

Davis, A., Marshak, A., Wiscombe, W. J., and Cahalan, R. F.: Scale-invariance of liquid water distributions in marine stratocumulus, i - spectral properties and stationarity issues, J. Atmos. Sci., 53, 1538-1558, 1996.

Davis, A. and Marshak, A.: Lévy kinetics in slab geometry: Scaling of transmission probability, in: Fractal Frontiers, (Eds) Novak, M. M. and Dewey, T. G., pp. 63-72, World Sci., N.J., 1998.

Funk, O.: Photon Pathlengths Distributions for Cloudy Skies Oxygen A-Band Measurements and Radiative Transfer Model Calculations, Ph.D. thesis, Institut für Umweltphysik, RuprechtKarls-Universität Heidelberg, 2000.

Gamache, R. R., Goldman, A., and Rothman, L. S.: Improved spectral parameters for the three most abundant isotopomers of the oxygen molecule, J. Quant. Spectr. Rad. Trans., 59, 495-509, 1998.

Harrison, L. and Min, Q.-L.: Photon path length distributions in cloudy atmospheres from ground-based high resolution $\mathrm{O}_{2}$ aband spectroscopy, in: IRS'96:Current Problems in Atmospheric Radiation, (Eds) Smith, W. L. and Stamnes, P., A. Deepak, Hampton, Va, 1997.

Isaacs, R. G., Wang, W.-C., Worsham, R. D., and Goldberg, S.: Multiple scattering lowtran and fascode models, Appl. Opt., 26, 1272-1281, 1987.

Marshak, A., Davis, A., Wiscombe, W., and Cahalan, R.: Radiative smoothing in fractal clouds, J. Geophys. Res., 100, 26247 $26261,1995$.

Min, Q., Harrison, L. C., and Clothiaux, E. E.; Joint statistics of photon path length and cloud optical depth: Case studies, J. Geophys. Res., 106, 7375-7385, 2001.

Moré, J., Garbow, B., and Hillstrom, K.: User guide for MINPACK1, Argonne National Labs Report ANL-80-74, Argonne, Illinois, 1980.

Nicolet, M.: On the molecular scattering in the terrestrial atmosphere: An empirical formula for its calculation in the homosphere, Planet. Space Sci., 32, 1467-1468, 1984.

Pfeilsticker, K.: First geometrical path lengths probability density function derivation of the skylight from spectroscopically highly resolving oxygen aband observations 2 . derivation of the lévyindex for the skylight transmitted by midlatitude clouds, J. Geophys. Res., 104, 4101-4116, 1999.

Pfeilsticker, K., Erle, F., Funk, O., Veitel, H., and Platt, U.: First geometrical path lengths probability density function derivation of the skylight from spectroscopically highly resolving oxygen aband observations 1 . measurement technique, atmospheric observations, and model calculations, J. Geophys. Res., 103, 11 483-11 504, 1998.

Portmann, R. W., Solomon, S., Sanders, R. W., and Daniel, J. S.: Cloud modulation of zenith sky oxygen photon path lengths over boulder, colorado: Measurements vs. model, J. Geophys. Res., 106, 1139-1155, 2001.

Ritter, K. J. and Wilkerson, T. D.: High resolution spectroscopy of the oxygen A-Band, J. Molec. Spectrosc., 121, 1-19, 1987.

Savigny, C. v., Funk, O., Platt, U., and Pfeilsticker, K.: Radiative smoothing in zenith-scattered skylight transmitted through optically thick clouds to the ground, Geophys. Res. Lett., 26, 29492952, 1999.

Slingo, A.: A gcm parameterization for the shortwave radiative properties of water clouds, J. Atm. Sci., 46, 1419-1427, 1989.

Stamnes, K., Tsay, S.-C., Wiscombe, W., and Jayaweera, K.: Numerically stable algorithm for discrete-ordinate-method radiative transfer in multiple scattering and emitting layered media, Applied Optics, 27, 2502-2509, 1988.

Stephens, G. L.: Optical Properties of Eight Water Cloud Types, CSIRO, tech. paper no. 36 edn., 1979.

Stephens, G. L. and Tsay, S.-C.: On the cloud absorption anomaly, Q. J. R. Meteorol. Soc., 116, 671-704, 1990.

Van de Hulst, H. C.: Multiple Light Scattering, Tables, Formulas and Applications, Volume 1 and 2, Academic Press, London, 1980.

Wild, M., Ohmura, A., Gilgen, H., and Roecker, E.: Validation of general circulation model radiative fluxes using surface observations, J. Climate, 8, 1309-1324, 1995.

Wild, M., Ohmura, A., Gilgen, H., Roecker, E., and Giogetta, M.: Improved representation of surface and atmospheric radiation budgets in the ECHAM4 general circulation model, MPIMeteorologie, Hamburg, report no. 200 edn., 1996. 